\title{
Electrical Characteristics of Near-Interface Traps in 3C-SiC Metal-Oxide-Semiconductor Capacitors
}

\author{
Fred C. J. Kong, Sima Dimitrijev, and Jisheng Han
}

\begin{abstract}
Deep-depletion capacitance measurements on n-type 3C-SiC MOS capacitors that exhibit both field and temperature dependencies are presented and analyzed in this letter. A two-step electron-detrapping process, in which electrons first tunnel from neutral near-interface traps to interface traps and are subsequently thermally emitted into the silicon carbide conduction band, is identified as the responsible mechanism. A mathematical model is proposed for this two-step detrapping process.
\end{abstract}

Index Terms-Capacitance transients, MOS capacitors, near-interface traps, $3 \mathrm{C}-\mathrm{SiC}$.

\section{INTRODUCTION}

3 C-SIC has an advantage over other $\mathrm{SiC}$ polytypes in that it can be integrated with silicon [1], [2]. With the ability to grow high-quality $\mathrm{SiO}_{2}$ layers by thermal oxidation, together with its wide energy gap, silicon carbide is well suited for the fabrication of MOS integrated circuits for operation in high-temperature environments. However, the high density of defects at the $\mathrm{SiO}_{2} / \mathrm{SiC}$ interface degrades the device performance and causes reliability problems [3], [4]. Traditionally, high-frequency capacitance-voltage $(\mathrm{HFC}-V)$ and capacitance transient $(C-t)$ techniques are used in the study of the deep-depletion capacitance relaxation of this interface [5]-[8]. Avila et al. observed an increase in the deep-depletion capacitance of 3C-SiC/Si MOS capacitors at room temperature and attributed this to the minority carrier generation mediated by interface traps [5]. In this mode of inversion layer formation, the generation rate is proportional to the intrinsic carrier concentration $n_{i}$ [9]. For 3C-SiC, $n_{i}$ is on the order of $10^{-1} \mathrm{~cm}^{-3}$ [10], compared to $10^{10} \mathrm{~cm}^{-3}$ in silicon. Given that the generation rate in $\mathrm{SiC}$ is 11 orders of magnitude smaller than that in silicon, we conclude that this generation process is practically nonexistent in $\mathrm{SiC}$ at room temperature. Tang et al. proposed that the observed increase in deep-depletion capacitance is due to minority carrier generation by impact ionization [8]. However, impact ionization is weakly temperature dependent [11], which is contrary to our observation that deep-depletion capacitance relaxation is strongly temperature dependent. As such, there is no satisfactory theory to correctly account for the capacitance relaxation.

Manuscript received January 16, 2008; revised June 11, 2008. This work was supported in part by an Australian Research Council under a Discovery Grant and in part by Qs Semiconductors. The work of J. Han was supported by the Australian Institute of Nuclear Science. The review of this letter was arranged by Editor S.-H. Ryu.

The authors are with the Queensland Microtechnology Facility and Griffith School of Engineering, Griffith University, Nathan, Qld. 4111, Australia.

Digital Object Identifier 10.1109/LED.2008.2001753

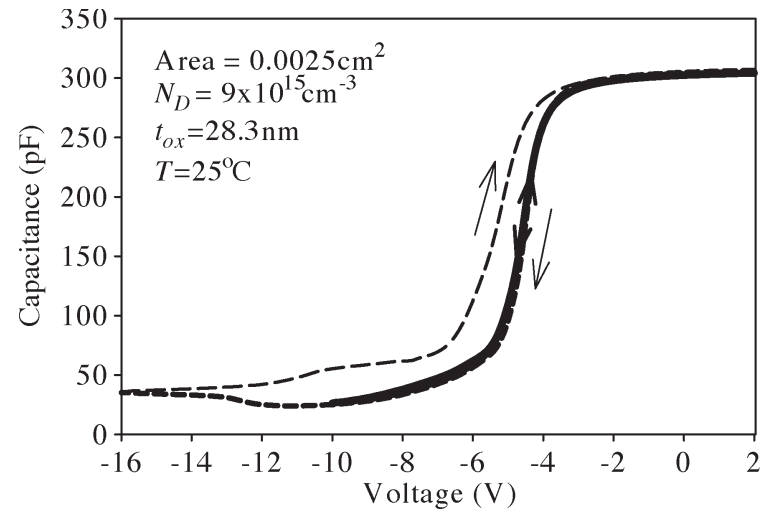

Fig. 1. Forward and reverse HFC $-V$ curves of a 3C-SiC MOS capacitor grown in $\mathrm{NO}(100 \%)$ at $1160{ }^{\circ} \mathrm{C}$ for two different bias sweeps measured at a frequency of $10 \mathrm{kHz}$ with a sweep rate of $0.1 \mathrm{~V} / \mathrm{s}$. The arrows show the directions of the bias sweeps.

In this letter, we combined the results of the measurements from $\mathrm{HFC}-V$ and $C-t$ to arrive at a new model for electron detrapping from near-interface traps at the $\mathrm{SiO}_{2} / \mathrm{SiC}$ interface, which accounts for the observed increase in deep-depletion capacitance.

\section{EXPERIMENT}

3C-SiC MOS capacitors were prepared on nitrogen-doped n-type $\left(10^{15}-10^{17} \mathrm{~cm}^{-3}\right) \mathrm{SiC}$ epitaxial films (with a thickness in the range of 4-6 $\mu \mathrm{m})$ supplied by NOVASiC, Institute of Crystal Growth (Berlin, Germany), and Hoya Advanced Semiconductor Technologies Co. Ltd. Gate oxides were grown in dry $\mathrm{O}_{2}$ or $\mathrm{NO}(100 \%)$ at $1160{ }^{\circ} \mathrm{C}$. The oxide thickness ranged from 18 to $23 \mathrm{~nm}$, as determined by accumulation capacitance measurements. The gate electrode is aluminum, with an area of $0.0025 \mathrm{~cm}^{2}$. The MOS capacitors are characterized by two different methods: 1) $\mathrm{HFC}-V$ measurement and 2) $C-t$ measurement, in which the capacitor is initially biased in accumulation and then pulsed to deep depletion and the capacitance is then measured as a function of time. All measurements were performed under a light-tight and electrically shielded environment.

\section{RESUlt AND Discussion}

Fig. 1 shows a typical $\mathrm{HF} C-V$ curve (solid lines) measured at room temperature when swept from 2 to $-10 \mathrm{~V}$ and vice versa. It can be seen that both the forward and reverse $C-V$ plots show deep depletion and are identical. The $C-V$ curves display a negative flatband voltage of $-4 \mathrm{~V}$ 


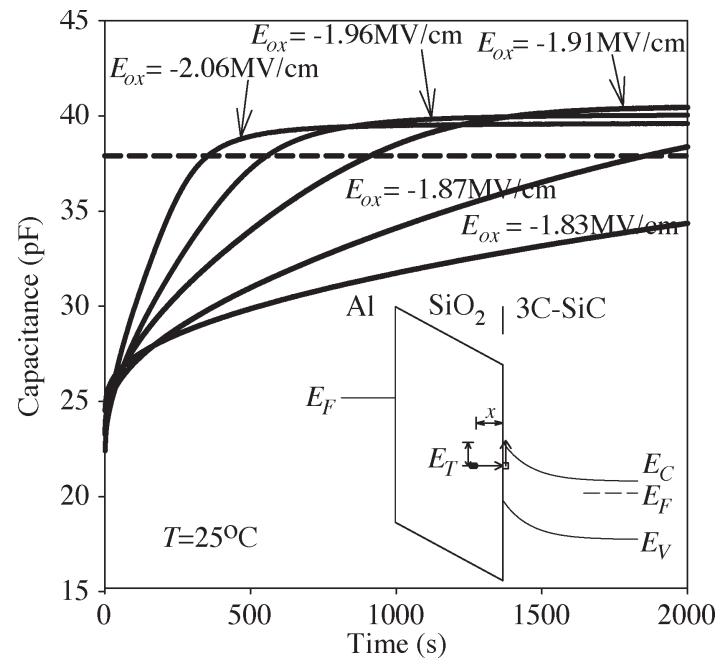

Fig. 2. Capacitance transients for five different oxide fields. The dotted line is the predicted inversion capacitance. The inset shows the two-step electrondetrapping process.

and, otherwise, minimal distortion when compared to the theoretical $C-V$ curve. The negative flatband voltage indicates that the trap density at or near the $\mathrm{SiO}_{2} / \mathrm{SiC}$ interface has an effective positive charge of $2.6 \times 10^{12} \mathrm{~cm}^{-2}$. The minimal distortion observed for these two curves indicates that the positive charge is due to traps that do not respond (emit or capture electrons) to the dc sweep in the applied bias range. When the dc negative bias is increased to $-16 \mathrm{~V}$, a hysteresis appears between the forward and reverse sweeps of the $\mathrm{HFC}-V$ curves, which are shown as dashed lines in Fig. 1. The reverse sweep exhibits a more negative flatband voltage, implying that a more positive charge is introduced, as a result of the higher negative bias. The positive trapped charge decreases as the bias is reduced, implying that the positive charge is being removed as the surface is being swept toward the accumulation. It is also noticed that the forward capacitance starts to increase at $-12 \mathrm{~V}$. This is attributed to an increase in the effective positive charge at or near the interface, which has the net effect of reducing the band bending, leading to the reduction in deep-depletion width and, therefore, the increase in capacitance. There is a hysteresis between the forward and reverse curves due to the fact that not all the traps that have emitted electrons between -10 and $-16 \mathrm{~V}$ can recapture the electrons before an accumulation bias is reached.

Fig. 2 shows the $C-t$ measurements at room temperature for different oxide field values (these are obtained by varying the gate bias) close to where the rise in deep-depletion capacitance started to occur in Fig. 1. If the deep-depletion capacitance relaxation is due to inversion carrier generation, all the curves will reach the same final steady-state inversion capacitance (shown as the dotted line in Fig. 2). It is clear that the final steady-state capacitances are above the predicted inversion capacitance. Furthermore, the capacitance transient is highly dependent on the oxide field. This is distinctly different from the capacitance transient due to the inversion carrier generation. We ascribe this to the electron detrapping from neutral nearinterface traps, which leaves behind a positive charge. In order to further investigate the detrapping process, we have developed a mathematical model to describe the field- and temperaturedependent capacitance transient. The distribution of the nearinterface traps per unit area at distance $x$ from the $\mathrm{SiC} / \mathrm{SiO}_{2}$ interface with a single energy level $E_{t}$, which was measured from the $\mathrm{SiC}$ conduction band, will be labeled $N_{T}\left(x, E_{t}\right)$. Labeling the corresponding detrapping time constant $\tau\left(x, E_{t}\right)$, the detrapping rate of the near-interface traps can be written as

$$
\frac{d N_{T}\left(x, E_{t}, t\right)}{d t}=-\frac{N_{T}\left(x, E_{t}, t\right)}{\tau\left(x, E_{t}\right)} .
$$

The rate of change of $N_{T}$ is also related to the rate of change of the total MOS capacitance $d C / d t$, using the relation [12]

$$
\frac{d N_{T}}{d t}=q N_{D} C_{\mathrm{ox}} \varepsilon_{\mathrm{SiC}} \frac{1}{C^{3}} \frac{d C}{d t}
$$

where $q$ is the electron charge, $N_{D}$ is the bulk dopant concentration, $\varepsilon_{\mathrm{SiC}}$ is the silicon carbide dielectric constant, and $C_{\mathrm{ox}}$ is the oxide capacitance per unit area. Equations (1) and (2) can then be integrated and combined to give the following equation:

$$
\frac{1}{C^{2}(t)}=\frac{2 N_{T}\left(t_{0}\right)}{N_{D} C_{\mathrm{ox}} \varepsilon_{\mathrm{SiC}}}\left(\exp (-t / \tau)-\exp \left(-t_{0} / \tau\right)\right)+\frac{1}{C^{2}\left(t_{0}\right)}
$$

where $N_{T}\left(t_{0}\right)$ is the initial trap density. Using (3), $\tau$ can be extracted from the measured $C(t)$ data in Fig. 2. As the extracted $\tau$ values shown in Fig. 3 exhibit both field and temperature dependencies, it points toward the two-step electrondetrapping process shown in the inset of Fig. 2 [13]. In this process, the electrons from the neutral near-interface traps tunnel to the interface traps, which are subsequently thermally emitted into the silicon carbide conduction band. This leaves positive charges in the near-interface traps that reduce the semiconductor field and thus cause capacitance relaxation. This is similar to the mechanism behind the transient threshold voltage shifts observed in the electrical stressing of MOSFETs [13]. It should be noted that there is no difference between repeated $C-V$ measurements after setting the MOS capacitor in accumulation. This indicates that the traps that become charged during the voltage sweep through deep depletion very quickly recapture electrons when the bias is set back to accumulation. There is an obvious asymmetry between the slow two-step electron-detrapping process and the very fast electron capture process. The reason for the faster capture process is the huge concentration of electrons in accumulation that are available for capturing by each trap.

Since the tunneling and thermal-emission steps occur in series, an effective first-order time constant for the two-step detrapping process can be written as [13]

$$
\tau\left(x, E_{t}\right)=\tau_{\text {tun }}\left(x, E_{t}\right)+\tau_{e}\left(E_{t}\right)
$$

The first term on the right-hand side of (4) represents the carrier tunneling time constant, in which the carrier tunnels from the near-interface traps at $E_{t}$ to an interface trap at the same energy 


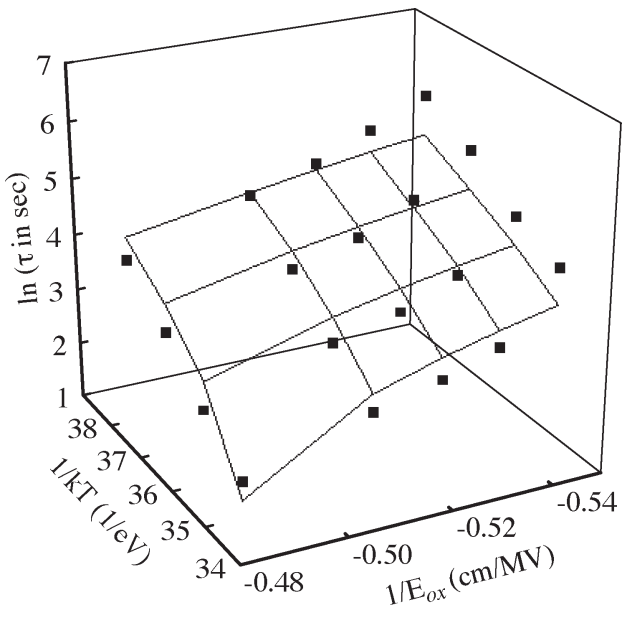

Fig. 3. Time constants as a function of oxide field and temperature. The measured temperature ranges from $30^{\circ} \mathrm{C}$ to $60{ }^{\circ} \mathrm{C}$. The 3-D plot shows (symbols) the experimentally extracted time constant and (grid) the best fit using (4).

level located at the $\mathrm{SiO}_{2} / \mathrm{SiC}$ interface through a trapezoidal potential barrier. This time constant is given by [13]-[15]

$\tau_{\text {tun }}\left(x, E_{t}\right)=\tau_{t} \exp \left(\frac{8 \pi \sqrt{2 m^{*}}}{3 h q E_{\text {ox }}}\left[\left(E_{t}\right)^{3 / 2}-\left(E_{t}-q E_{\text {ox }} x\right)^{3 / 2}\right]\right)$

where $\tau_{t}$ is a fitting constant, $h$ is the Planck's constant, $q$ is the electron charge, $m^{*}$ is the effective tunneling mass for electrons in the oxide, and $E_{\mathrm{ox}}$ is the oxide field. The second term in (4) describes the thermal emission time constant for the carrier at interface traps emitted to the silicon carbide conduction band and is given by [9]

$$
\tau_{e}\left(E_{t}\right)=\tau_{o} \exp \frac{\left(E_{C}-E_{t}\right)}{k T}
$$

where $\tau_{o}$ is a preexponential constant, and $E_{C}$ is the energy at the bottom of the silicon carbide conduction band. By fitting (4)-(6) to the measured time constants shown in Fig. 3, the tunneling distance and the energy level of the near-interface traps are found to be $0.7 \mathrm{~nm}$ and $0.40 \mathrm{eV}$ below the conduction band edge, respectively. The extracted near-interface trap tunneling distance is comparable to that extracted from the transient voltage shift relaxation method for the $\mathrm{SiO}_{2} / \mathrm{Si}$ interface [13]. It is most likely that there are active nearinterface traps at different energy levels and distances from the interface. Therefore, the extracted $x$ and $E_{t}$ are simply the average values for all these traps.

\section{CONCLUSION}

In summary, we have shown that the electron detrapping from neutral near-interface traps is responsible for the rise in capacitance in the deep-depletion region and the hysteresis effect of the $\mathrm{HFC}-V$ curves for $3 \mathrm{C}-\mathrm{SiC}$ MOS capacitors. We have also established a new model that provides close agreement with the 3C-SiC MOS capacitance behavior experimentally observed. The presented experimental results and model show that inversion carrier generation is not required to account for the rise in capacitance at high deep-depletion bias.

\section{REFERENCES}

[1] S. Nishino, J. A. Powell, and H. A. Will, "Production of large-area singlecrystal wafers of cubic $\mathrm{SiC}$ for semiconductor devices," Appl. Phys. Lett., vol. 42, no. 5, pp. 460-462, Mar. 1983.

[2] H. Nagasawa and K. Yagi, "3C-SiC single-crystal films grown on 6-inch Si substrates," Phys. Stat. Sol. (B), vol. 202, no. 1, pp. 335-358, Jul. 1997.

[3] V. V. Afanas'ev, F. Ciobanu, G. Pensl, and A. Stesmans, Recent Major Advances in SiC, W. J. Choyke, H. Matsunami, and G. Pensl, Eds. New York: Springer-Verlag, 2003, pp. 343-371.

[4] P. Jamet, S. Dimitrijev, and P. Tanner, "Effects of nitridation in gate oxides grown on 4H-SiC," J. Appl. Phys., vol. 90, no. 10, pp. 5058-5063, Nov. 2001.

[5] R. E. Avila, J. J. Kopanski, and C. D. Fung, "Behavior of inversion layers in 3C silicon carbide," Appl. Phys. Lett., vol. 49, no. 6, pp. 334-336, Aug. 1986.

[6] S. Zaima, K. Onoda, Y. Koide, and Y. Yasuda, "Effects of oxidation conditions on electrical properties of $\mathrm{SiC}-\mathrm{SiO}_{2}$ interfaces," J. Appl. Phys., vol. 68, no. 12, pp. 6304-6308, Dec. 1990.

[7] M. Shinohara, M. Yamanaka, S. Misawa, H. Okumura, and S. Yoshida, " $C-V$ characteristics of MOS structures fabricated of Al-doped p-type 3C-SiC epilayers grown on Si by chemical vapor deposition," Jpn. J. Appl. Phys., vol. 30, no. 2, pp. 240-243, Feb. 1991.

[8] S. M. Tang, W. B. Berry, R. Kwor, M. V. Zeller, and L. G. Matus, "High frequency capacitance-voltage characteristics of thermally grown $\mathrm{SiO}_{2}$ films on $\beta$-SiC," J. Electrochem. Soc., vol. 137, no. 1, pp. 221-225, Jan. 1990.

[9] W. Shockley and W. T. Read, "Statistics of the recombinations of holes and electrons," Phys. Rev., vol. 87, no. 5, pp. 835-842, Sep. 1952.

[10] M. E. Levinshtein, S. L. Rumyantsev, and M. S. Shur, Properties of Advanced Semiconductor Materials GaN, AlN, SiC, BN, SiC, SiGe. New York: Wiley, 2001, pp. 93-148.

[11] S. M. Sze, Physics of Semiconductor Devices. New York: Wiley, 1981, ch. 1 .

[12] D. K. Schroder, Semiconductor Material and Device Characterization. New York: Wiley, 2006, ch. 5.

[13] T. L. Tewksbury, III and H. S. Lee, "Characterization, modeling, and minimization of transient threshold voltage shifts in MOSFETs," IEEE J. Solid-State Circuits, vol. 29, no. 3, pp. 239-252, Mar. 1994.

[14] T. K. Kang, M. J. Chen, C. H. Liu, and S. K. Fan, "Numerical confirmation of inelastic trap-assisted tunneling (ITAT) as SILC mechanism," IEEE Trans. Electron Devices, vol. 48, no. 10, pp. 2317-2322, Oct. 2001.

[15] T. Wang, L. P. Chiang, N. K. Zous, T. E. Chang, and C. Huang, "Characterization of various stress-induced oxide traps in MOSFETs by using a subthreshold transient current technique," IEEE Trans. Electron Devices, vol. 45, no. 8, pp. 1196-1791, Aug. 1998. 